\title{
Automatic 3D visualization and paper pop-up parts fabrication of construction model by recognizing 2D image of floor plan (Withdrawal Notice)
}

Yuho Nobuhara, Shugo Nishioka, Toru Matsumoto, Kunio Sakamoto

Yuho Nobuhara, Shugo Nishioka, Toru Matsumoto, Kunio Sakamoto, "Automatic 3D visualization and paper pop-up parts fabrication of construction model by recognizing 2D image of floor plan (Withdrawal Notice)," Proc. SPIE 10819, Optical Metrology and Inspection for Industrial Applications V, 108191B (2 November 2018); doi: 10.1117/12.2500019

SPIE. Event: SPIE/COS Photonics Asia, 2018, Beijing, China 


\section{Automatic 3D visualization and paper pop-up parts fabrication of construction model by recognizing 2D image of floor plan (Withdrawal Notice)}

Yuho Nobuhara, Shugo Nishioka, Toru Matsumoto, Kunio Sakamoto

Konan Univ. (Japan)

Proc. SPIE 10819, 108191B (2018)

Online Publication Date: 2 November 2018

Withdrawn from Publication: 4 February 2019

Conference Date: 11 October-13 October 2018

Conference Location: Beijing, China

Conference Title: Optical Metrology and Inspection for Industrial Applications V

Conference Chairs: Sen Han, Toru Yoshizawa, Song Zhang

Publisher's Note: This manuscript, originally published on 2 November 2018, has been withdrawn by the publisher for editorial reasons. 